\title{
The Modeling and Simulation of WSN Triangular Array Sound Source Localization Method
}

\author{
Jing Tian \\ College of Computer Science and Technology \\ Jilin University \\ Changchun Jilin 130012, China
}

\author{
Yinghui Cao* \\ College of Computer Science and Technology \\ Jilin University \\ Changchun Jilin 130012, China \\ *Corresponding author
}

\author{
Chengquan $\mathrm{Hu}$ \\ College of Computer Science and Technology \\ Jilin University \\ Changchun Jilin 130012, China
}

\begin{abstract}
The sound source localization method that based on triangular array of wireless sensor networks (WSN) is proposed to locate the sound source and research the error distribution. Using the Time Difference of Arrival (TDOA) algorithm, the proposed sound source localization method can be used to locate the source of impulse of sound inside or around the WSN array. The simulation experiment had positioning error distribution. Simulation results show that, the effective localization region of the proposed method is mainly located inside the WSN array and extents outward across the edges of the array. The effective localization region became bigger with the WSN array scale became lager. The simulation results also show that influence of the error of WSN node position to the localization error is greater than the errors of sound arrival delay calculation. The influence of sound arrival time difference analysis error and node location error for the effect of sound source localization increased with the increase of the WSN array scale.
\end{abstract}

Keywords-wireless sensor network(WSN); sound source localization; time difference of arrival (TDOA); simulation; Error analysis

\section{INTRODUCTION}

At present, the sound source localization technology based on sensor nodes has a broad application, mainly manifested in two aspects: security, such as abnormal sound source localization in public places [1], bank security monitoring, measurement technique of playing point positioning system design and implementation based on the time delay [2], etc. On the other hand is sound detection, such as tracking camera system based on sound source localization, the algorithm of gearbox noise source localization based on microphone array studies [3], etc.

Commonly sound source localization method can be divided into three categories [4]: localization method based on beam forming, positioning method based on high resolution spectrum estimation and based on signal to the time difference method. In this paper using signals to reach the time difference method (TDOA) [5].Sound signal from the source through time of arrival in different sound sensor general could use generalized cross correlation, GCC[6], cross - power spectrum phase, CSP [7] .

At present, mainly the research work on small sensors array to source position of sound source, and how to improve the noise environment in a certain sound to reach the precision of the time difference and anti-interference ability. In this paper, based on the triangle array of wireless sensor network (WSN), a sound source localization method for simulation modeling, and the method of sound source localization error in numerical calculation, analysis of different error sources (including voice to the analysis of the jet lag error and WSN node position) to the effect of sound source localization error, as well as the effective positioning range distribution of numerical analysis. In addition, this paper puts forward the sound source localization method used in the wireless sensor node transmission of voice signal collection, don't need cables being laid, has the characteristics of good flexibility and easy deployment.

\section{SOUND SOURCE LOCALIZATION METHOD AND THE SIMULATION PROCESS}

\section{A. Sound Source Localization Method}

This paper adopts the WSN triangle array .WSN nodes by GSP module itself can be positioning and timing. WSN nodes by wireless communication to gather voice signal transmission to the computer, calculated by the computer through signal cross-correlation algorithm, sound pulse signals (such as explosions, gunfire, etc) in different WSN node to the time difference, then calculated according to the sound source location TODA method.

Figure 1 shows the calculation principle diagram. P1, P2, P3 is WSN node, locate in $(\mathrm{x} 1, \mathrm{y} 1),(\mathrm{x} 2, \mathrm{y} 2),(\mathrm{x} 3$, $\mathrm{y} 3)$. The location of sound source $\mathrm{P}$ is $(\mathrm{x}, \mathrm{y})$, the distance to node $\mathrm{i}$ is $L_{i}, L_{i}=\sqrt{\left(\mathrm{x}-x_{i}\right)^{2}+\left(y-y_{i}\right)^{2}}$ the distance 
difference to node $\mathrm{i}, \mathrm{j}$ is $\Delta L_{i j}=L_{i}-L_{j}, \quad$ and $i, j \in(1,2,3), i \neq j$. The time of node $\mathrm{i}$ get the sound is $t_{i}$, the time difference of node $\mathrm{i}, \mathrm{j}$ is $\Delta t_{i j}=t_{i}-t_{j}$, the equation based on TDOA is

$$
\Delta L_{i j}=\Delta t_{i j} \cdot v
$$

$\mathrm{V}$ is sound speed. Get the time difference $\Delta t_{i j}$ and the WSN node location $P_{i}\left(x_{i}, y_{i}\right)$, through equation(1) can calculate the sound source location.

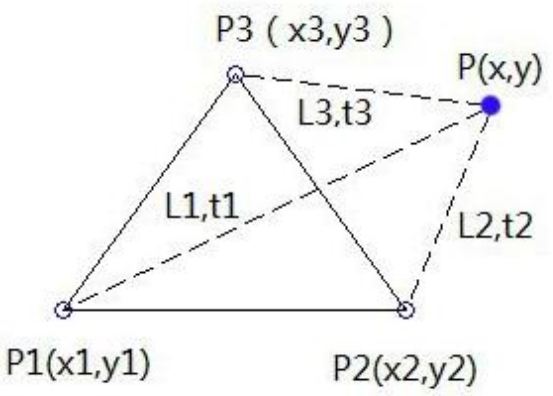

\section{FIGURE I. SOUND SOURCE LOCALIZATION CALCULATION PRINCIPLE DIAGRAM}

\section{B. Simulation Process}

In WSN triangle array simulation process, first calculate source to the WSN nodes time difference $\Delta t_{i j}$, based on TDOA method use $\Delta t_{i j}$ and WSN nodes' position $P_{i}\left(x_{i}, y_{i}\right)$ calculate the sound source location.

Due to the actual location in the process of the signal arrival time difference analysis of WSN node calculation, and the positioning of the WSN node itself has certain error, the calculation also has a certain error. In order to analyze the sound source position error, the simulation has the error of time difference between each node and the WSN node location, then solve the equations (1) to get the sound source location, and calculate the error source.

The simulation process steps:

1) In a certain range scan the sound source location $P(x, y)$, and calculate the time difference $\Delta t_{i j}$ between the WSN node;

2) Import a certain range of the time difference error of $\Delta t_{i j}$ and WSN node location error. Calculate equations (1) to get the sound source location, and the sound source localization error err $=\sqrt{\left(x^{\prime}-x\right)^{2}-\left(y^{\prime}-y\right)^{2}} r$;
3) For the specific location of sound source, repeat positioning calculation (step 2) thirty times, then the average of sound source localization error, and then continue to scan the sound source location.

\section{THE SimUlation EXPERIMENT AND RESUlt ANALYSIS}

This section according to the simulation steps are described in the section 2.2, the time difference $\Delta t_{i j}$ and the WSN node location $P_{i}\left(x_{i}, y_{i}\right)$ has a certain range of random error.

For the gunfire and explosions pulse sound source, use the signal cross-correlation calculation method, and take more than $10 \mathrm{KHZ}$ sampling rate, make the error of time difference only 1 millisecond. In this paper, import $\pm 5 \mathrm{~ms}, \pm 2 \mathrm{~ms}, \pm 1 \mathrm{~ms}$ time difference error .For WSN node using GPS positioning module, the error usually about $\pm 5 \mathrm{~m}$.In this paper import $\pm 5 \mathrm{~m}, \pm 2 \mathrm{~m}, \pm 1 \mathrm{~m}$ location error to the WSN node.

\section{A. The Influence of Node Location Error to Position}

First, assume that there is no time difference error, In order to show different levels of nodes locate error's influence to position, use $100 \mathrm{~m}$ of WSN triangle array to calculate the locate error with the node's position error of $\pm 5 \mathrm{~m}, \pm 2 \mathrm{~m}$, $\pm 1 \mathrm{~m}$.

For the simulation calculation results, when the sound source position error in the range of $0 \sim 50$ meters with blue and red linear mapping, display more than $50 \mathrm{~m}$ in gray.

Figure 2 (a) shows the node location error range in $\pm 5 \mathrm{~m}$. The effective range is the inside of a triangular array and from the center of the array trilateral array external extension area of about $50 \mathrm{~m}$.

Figure 2 (b) shows the node location error range in $\pm 2 \mathrm{~m}$. The effective range is the inside of a triangular array and from the center of the array trilateral array external extension area of about $190 \mathrm{~m}$. Compared with figure 2 (a),the effective range is lager.

Figure 2(c) shows the node location error range in $\pm 1 \mathrm{~m}$. The effective range is similar with Figure 2 (b), and lager than Figure 2(a).

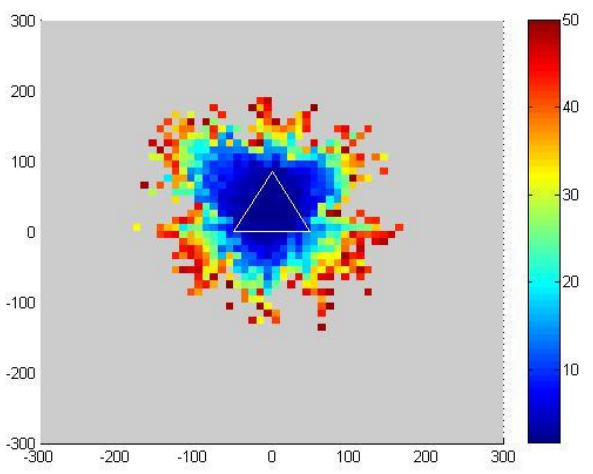

(a) The node locate error range in $\pm 5 \mathrm{~m}$ 


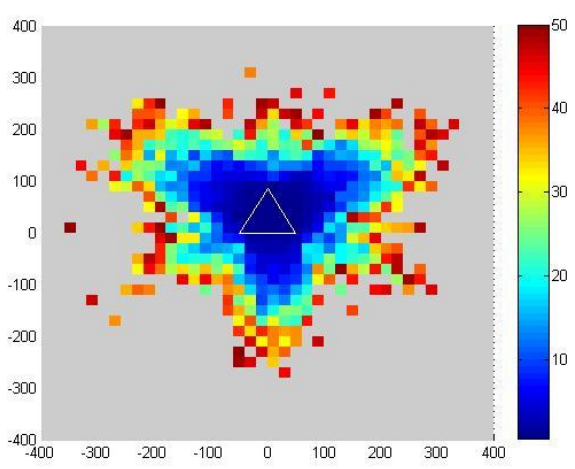

(b) The node locate error range in $\pm 2 \mathrm{~m}$

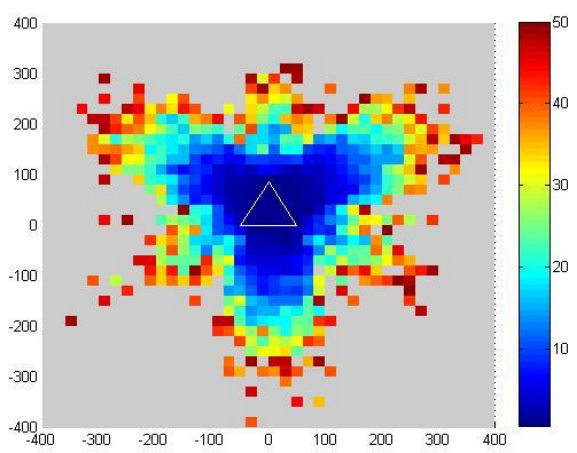

(c) The node locate error range in $\pm 1 \mathrm{~m}$

FIGURE II. THE ERROR DISTRIBUTION OF $100 \mathrm{M}$ WSN TRIANGLE ARRAY WITH NODE POSITION ERROR.

\section{B. The Influence of Time Difference Error to Position}

First, assume that there is no nodes locate error, In order to show different levels of time difference error's influence to position, use $100 \mathrm{~m}$ of WSN triangle array to calculate the locate error with the time difference error of $\pm 5 \mathrm{~ms}, \pm 2 \mathrm{~ms}$, $\pm 1 \mathrm{~ms}$..

Figure 3 (a) shows the time difference error range in $\pm 5 \mathrm{~ms}$. The effective range is the inside of a triangular array and from the center of the array trilateral array external extension area of about $100 \mathrm{~m}$.

Figure 3 (b) shows the time difference error range in $\pm 2 \mathrm{~ms}$. The effective range is the inside of a triangular array and from the center of the array trilateral array external extension area of about $300 \mathrm{~m}$. And has about $50 \mathrm{~m}$ extension outward along the three vertices .Compared with figure 2 (a), the effective range is lager. And has extension outwards the vertices.

Figure 3(c) shows the time difference error range in $\pm 1 \mathrm{~ms}$. The effective range is similar with figure 3 (b), but the area of error under $50 \mathrm{~m}$ is lager the figure 3 (b).

Compare with section 3.1 , the range of $\pm 5 \mathrm{~m}$ node position error influence on calculation accuracy is greater than the influence of $\pm 5 \mathrm{~ms}$ time difference error. So the locate system need more accuracy of the nodes positioning, then in the sound source localization system should try to improve the positioning precision of the WSN nodes.

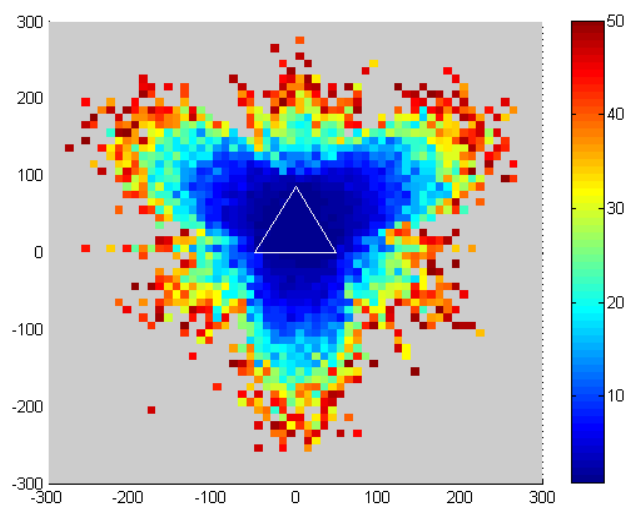

(a) Time difference error range in $\pm 5 \mathrm{~ms}$

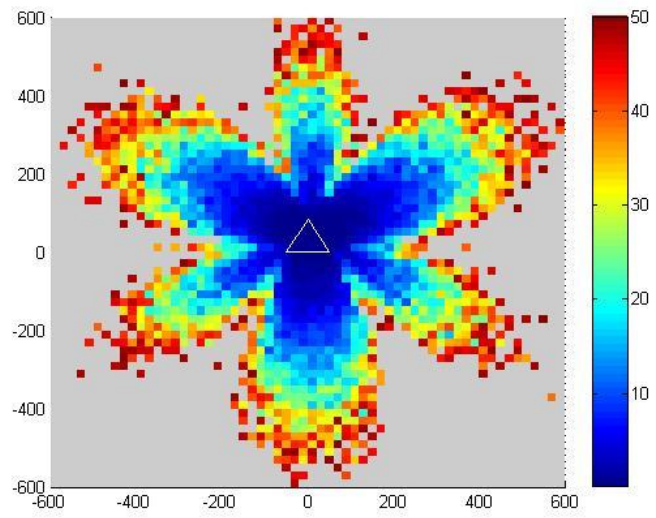

(b) Time difference error range in $\pm 2 \mathrm{~ms}$

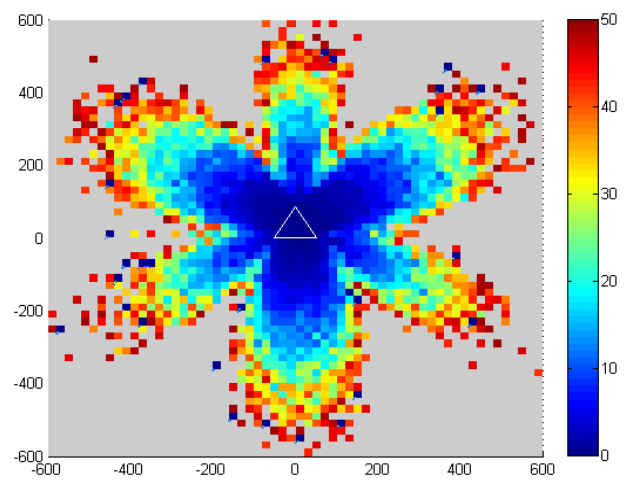

(c) Time difference error range in $\pm 1 \mathrm{~ms}$

FIGURE III. THE ERROR DISTRIBUTION OF $100 \mathrm{M}$ WSN TRIANGLE ARRAY WITH TIME DIFFERENCE ERROR

\section{The Influence of Time Difference Error to Position}

In order to study the influence of different array shapes on the effective range, the simulation experiments of three kinds of triangle array are carried out. Figure 4 (a) is a triangular array, figure 4 (b) array's high is half of (a), figure 4 (c) array's bottom length is half of (a). To show the distribution of 
sound source localization, import $\pm 5 \mathrm{~ms}$ range of time difference error to the three kinds of array.

Figure 4 (a) shows the effective range is the inside of a triangular array and from the center of the array trilateral array external extension area of about $100 \mathrm{~m}$.

Figure 4 (b) shows effective range is the inside of a triangular array, and effective range extension in the direction of $\mathrm{Y}$ axis is $100 \mathrm{~m}$ expansion, but in the other direction expansion less error value is higher, close to the red. In comparison with Figure 4 (a), the effective range of the array can be seen in the range of (b), and the effective range of the array is less than (a).

Figure 4 (c) shows the effective sound source localization range in the triangular array and effective range extension in the direction of $\mathrm{X}$ axis is about $100 \mathrm{~m}$ extended, but the other direction expansion less error value is higher, close to the red. Compared to (a), the expansion of the effective range of (b) and (c) is smaller.

The sound source location error of three kinds of shapes is calculated. The ratio of the effect area and the area of the triangle are calculated. The value of a positive triangle is 2.8 , and the other shapes are 2.4 and 2.3. In the case of the same size of the same array, the effective range of the sound source localization system is more extensive.

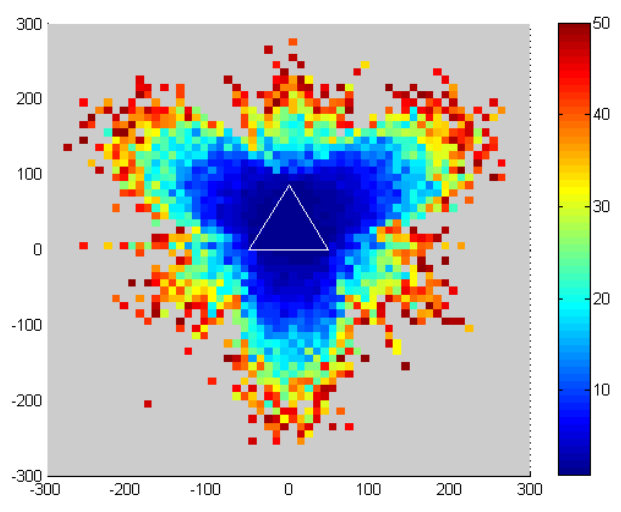

(a) Positive triangle array

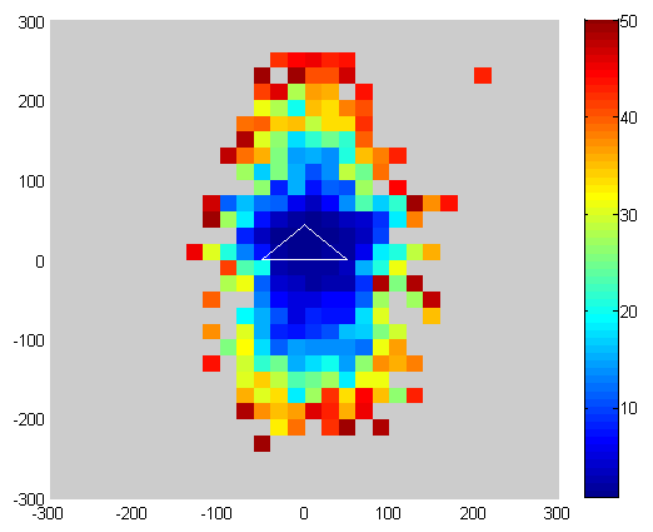

(b)High half of positive triangle array

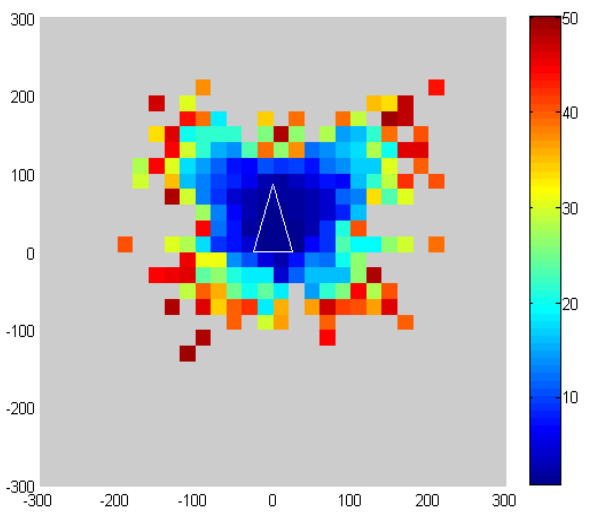

(c) Bottom length half of positive triangle array

FIGURE IV. THE EFFECT RANGE OFTHREE KIND OF TRIANGLE ARRAY

\section{SUMMARY}

This paper is based on WSN .Use three sensor nodes to locate sound source. The simulation result:

1) The effective range mainly in the triangle array, and extended along the array edge to array external to a certain extent, the extension area increases with the array size;

2) In the system, import time difference error and node position error, the larger the size of the array, the smaller the influence of the error, the location more effect. The same size of the array, the smaller the error, the more accurate positioning;

3) In the system, the node position error has more influence than time difference error; under the similar array size and the error condition, the effective range of a positive triangle array is larger than other shapes.

\section{REFERENCES}

[1] Hongwei Li, Haibing Tang, Weiguo Gong.Time delay estimation method of abnormal sound source localization in public places[J]. Chinese Journal of Scientific Instrument, 2012, 33(4): 750-756.

[2] Jianshen Ye. Design and Implementation of Projectile Falling Point Localization System Based on Acoustic Time-Delay Measure Technology[D]. TaiYuan:Master degree of North Central University, 2007:15-26.

[3] Shi Quan, Dong Guo, Xiaohui Shi. Automotive transmission noise source identification based on microphone array.[J]. JOURNAL OF VIBRATION AND SHOCK, 2012, 31(13): 134-137.

[4] BRANDSTEIN M. Microphone Arrays[M].New York: Springer-Verlag, 2001.

[5] Guoqing Zhao.. Radar countermeasure principle. XiAn,Xi'an Electronic and Science University press. $1999: 63 \sim 67$

[6] KNAPP C H, CARTER G C. The generalized correlation method for estimation of time delay [J] . IEEE Transactions on Acoustics, Speech, and Signal Processing, 1976, 24 ( 4) : 320-327.

[7] OMOLOGO M, SVAIZER P. Acoustic source location in noisy and reverberant environment using CSP analysis [C] / /IEEE International Conference on Acoustics, Speech, and Signal Processing. Atlanta, USA, 1996: 921- 924. 